$\underline{\text { Preprint typeset in JHEP style - HYPER VERSION }}$

hep-th/0605263

UCB-PTH-06/10

LBNL-60251

\title{
Holographic probabilities in eternal inflation
}

\author{
Raphael Bousso \\ Center for Theoretical Physics, Department of Physics \\ University of California, Berkeley, CA 94720-7300, U.S.A. \\ and \\ Lawrence Berkeley National Laboratory, Berkeley, CA 94720-8162, U.S.A. \\ E-mail: bousso (at) lbl.gov
}

\begin{abstract}
The problem of assigning probabilities to vacua is notoriously ambiguous in the global description of eternal inflation. The local point of view is preferred by holography, and it naturally picks out a simple probability measure. This requires no ambiguous choices, such as which time slices to use, or how to weight the volume occupied by a vacuum. The local viewpoint also cuts off the weight carried by exponentially large slow-roll expansion factors or lifetimes.
\end{abstract}




\section{Contents}

1. Introduction 1

2. Prior probabilities 3

2.1 Preliminaries 3

2.2 Graph formulation 4

2.3 A simple example

2.4 Convergence and pruned trees 6

2.5 A cyclic example 8

2.6 Matrix formulation 9

3. Weighting 11

\section{Introduction}

In a theory with multiple metastable vacua, such as string theory $[1,2]$, the prediction of low-energy parameters necessarily involves a statistical element. Part of the challenge is to survey all the vacua in the landscape $[3,4]$. Another important part is to understand how cosmological dynamics selects among them.

This dynamics will be eternal inflation driven by the false vacua with positive cosmological constant. But the task of assigning probabilities in eternal inflation is plagued by ambiguities [5]. Should vacuum $i$ be weighted by the number of $i$-bubbles, or by the volume that they occupy? And at what time do we compare these numbers or volumes? There is no preferred time slicing in eternal inflation.

A number of interesting probability measures have been proposed, most recently in Refs. [6-8]. The fact that they give different answers illustrates just how difficult this problem is. One can imagine other prescriptions, and it appears that practically any answer can be obtained by devising a suitable time slicing.

Most proposals have been formulated in terms of a global geometry (see however Ref. [9]), which involves an infinite number of causally disconnected regions that can never communicate. This picture was always at best a convenient fiction-and the above ambiguities make it look rather inconvenient. 
The purpose of this paper is to develop a probability measure by appealing only to a single, causally connected region, or causal diamond [10]. This is called the local, or causal, or holographic point of view. There are two specific reasons to embrace this viewpoint.

From the quantum properties of black holes, we have learned that the simultaneous description of two causally disconnected regions leads to paradoxes, which are resolved if we stick to describing only what any one observer can measure [11].

More recent arguments [12] show that the situation in eternal inflation is worse than for black holes. An observer outside a black hole can compute the interior geometry from initial conditions, but an observer in eternal inflation cannot predict when and where bubbles will form, and so cannot distinguish between macroscopically distinct global metrics [12]. Thus, the local observer cannot even construct a global geometry that one then could argue about how to slice.

The former argument shows that the local point of view is theoretically preferred; the latter encourages us that it will not simply mirror the ambiguities that plague the global description. Indeed, we will find that the local computation of probabilities involves none of the arbitrary choices that abound in the global approach.

In the local prescription, we consider only a single worldline. The task of computing the probability of a vacuum then breaks up into two separate problems.

1. How likely is it for the worldline to enter vacuum $i$ ?

2. What is the probability that observers will emerge in vacuum $i$ ?

The quantitative results of this paper pertain mainly to the first question. They are presented in Sec. 2. The holographic point of view also has surprising implications for the second question, which we discuss in Sec. 3 .

Relation to other work Though we take a very different starting point, we will employ much of the elegant and powerful machinery devised in other approaches to the measure problem. Easther et al. [8] follow not one, but an infinite number of worldlines sprinkled on the global geometry. Their measure is essentially equivalent to an earlier proposal by Garriga et al. [7], which is formulated in a matrix language. The relative decay probability $\eta_{i j}$, which is at the heart of our prescription, has recently appeared prominently in Ref. [13] (where it is called transition matrix, $T_{i j}$ ).

We will arrive at a measure that is, as far as we are aware, strictly inequivalent to all previous proposals. It differs from Ref. [6], for example in that metastable vacua generally have nonzero probability (an reassuring feature given the evidence that our 
vacuum is metastable). It is also inequivalent to Refs. $[7,8]$, for example in that it can depend on initial conditions. ${ }^{1}$

One can consider "cyclic" landscapes, in which all vacua are metastable (though this appears to be empirically ruled out [14]). By comparing our result to Ref. [13], we shall see that our measure, and the measure of Ref. [7], reduce to the same prescription in this special case.

\section{Prior probabilities}

In this section, we find the probability $p_{i}$ for a single, fixed worldline to enter vacuum $i$. Every time a vacuum bubble is nucleated, free energy can be produced, and observers may emerge as the region relaxes to equilibrium (see Sec. 3). Hence, the vacuum $i$ is more favored if the worldline enters it more than once. A probability measure reflecting this can be computed using tree graphs. We will first give a particularly straightforward prescription, which has redundancies that can lead to divergences. We find that it can be considerably streamlined, and the resulting "pruned tree" prescription has no divergences. Finally, we will derive a simple matrix equation for the probability vector p.

\subsection{Preliminaries}

Consider a landscape with vacua $i$. We will assume that this includes metastable vacua, which eventually decay into other vacua. There may also be "terminal" vacua, which do not decay. If so, we call the landscape terminal; otherwise we call it cyclic. ${ }^{2}$

Our prescription will be general enough to deal with both types of landscapes. However, the former case is physically more interesting. Empirically, there is evidence that terminal vacua are present in the landscape $[14,15]$. Moreover, the landscape of string theory is terminal.

For simplicity, let us assume that the landscape is connected. By this we mean that every vacuum can be reached with nonzero probability from any metastable vacuum by some sequence of decays. (Otherwise, our prescription can still be applied, with the relative probability for each connected part of the landscape determined solely by initial conditions.)

Let $\kappa_{i j}$ be the probability per unit proper time for a geodesic wordline in vacuum $j$ to enter vacuum $i$. The expected amount of time a worldline will spend in the vacuum $j$ is $\left(\sum_{i} \kappa_{i j}\right)^{-1}$. For a terminal vacuum $z, \kappa_{i z}=0$ for all $i$.

\footnotetext{
${ }^{1}$ Some authors regard this as undesirable, but I can see no physical reason why. A theory of initial conditions is needed in any case to understand why the universe did not start in a true vacuum.

${ }^{2}$ We will not consider slow-roll eternal inflation here, though similar methods can be applied.
} 
For the purposes of computing prior probabilities, we will be interested not in how long the worldline lingers in a metastable vacuum $a$, but only which vacuum it enters next. This is described by the relative probability to decay to $i$,

$$
\mu_{i a}=\frac{\kappa_{i a}}{\sum_{j} \kappa_{j a}}
$$

In matrix language, $\mu$ arises by normalizing each column of $\kappa$ to sum to 1 (except for columns corresponding to terminal vacua, which are all zero). Further properties of this matrix will be discussed in a future publication.

Our prescription cares only about relative decay rates between vacua. The value of the cosmological constant does not enter directly. However, it is worth recalling that there is a close relationship between metastability and the sign of the cosmological constant.

Let us first consider de Sitter vacua, which have $\Lambda>0$. The entropy of de Sitter space is $3 \pi / \Lambda$, so the phase space is finite. Since we assume that the landscape is connected, there exists at least one decay channel that produces the vacuum in question. Then the reverse channel cannot be suppressed by an extra factor smaller than $e^{-3 \pi / \Lambda}$, which is nonzero. Hence, vacua with $\Lambda>0$ are necessarily metastable.

Vacua with $\Lambda=0$ are asymptotically locally supersymmetric - why else would the cosmological constant vanish exactly? Hence one would expect that $\Lambda=0$ vacua are stable. $^{3}$ Vacua with $\Lambda<0$ end in a big crunch after a time of order $\Lambda^{-1 / 2}$. Hence they are also terminal, unless they are so unstable that the (generically exponentially long) decay time is shorter than $\Lambda^{-1 / 2}$.

\subsection{Graph formulation}

Suppose the worldline starts out in vacuum $o$. If this is terminal, there won't be anything to compute, so let's assume that it is metastable. We will later generalize this initial condition to a probability distribution over several initial vacua.

The most straightforward way to compute probabilities is to draw a tree that begins with a root node labeled $o$. For each vacuum $i$ that $o$ can decay into, draw a branch connecting $o$ with a new node labeled with the new vacuum $i$. Next to each branch, write the relative probability, $\mu_{\text {branch }}$, for this decay channel (in this case, $\mu_{\text {branch }}=\mu_{i o}$ ).

\footnotetext{
${ }^{3}$ I thank E. Silverstein for emphasizing to me that this is not obvious. Decay into a $\Lambda=0$ vacuum produces an open FRW universe with finite energy density and infinite total energy. Globally, this is not a supersymmetric state, nor even a finite energy state, so there is no rigorous argument for stability. There are SUSY-independent obstructions against jumping from zero to positive cosmological constant $[16,17]$. But one could imagine a kind of stimulated decay [18] producing negative energy bubbles.
} 
Then repeat this procedure for each metastable new vacuum. This will generate a tree. (We will later show that this tree can be simplified considerably.)

Next, we compute a raw (i.e., unnormalized) probability for each vacuum in the landscape. For each path from the root node to the vacuum in question, multiply the branch probabilities; then sum up the results:

$$
P_{i}=\sum_{\begin{array}{c}
\text { all nodes } \\
\text { labelled } i
\end{array}} \prod_{\begin{array}{c}
\text { the branches } \\
\text { forming the path } \\
\text { from the root to the node }
\end{array}} \mu_{\text {branch }}
$$

From the raw probabilities the normalized prior probabilities can be easily computed:

$$
p_{i}=\frac{P_{i}}{\sum_{j} P_{j}} .
$$

This is the probability for a worldline to pass through vacuum $i$.

\subsection{A simple example}

Consider a landscape with two metastable vacua $A$ and $B$, and a terminal vacuum $Z$, as shown in Fig. 1. In this model, $A$ can only decay to $B\left(\eta_{B A}=1\right)$. The vacuum $B$ decays to $Z$ with probability $\eta_{Z B}=1-\epsilon$, or back up to $A$ with probability $\eta_{A B}=\epsilon$. We will not assume that $\epsilon$ is small. Physically one expects $\epsilon \ll 1$ if the cosmological constant of $A$ is larger than that of $B$, as shown in the potential in the center of Fig. 1. However, the computations below will be unchanged if $A$ lies below $B$, in which case $\epsilon$ could be anything.

First, let us suppose that the initial vacuum is $A$. From the associated tree (Fig. 1, left), one sees that there are infinitely many paths leading into each vacuum. For vacuum $A$, the paths are are $A B A, A B A B A, \ldots$, giving a raw probability $P_{A}=$ $\epsilon+\epsilon^{2}+\ldots=\epsilon /(1-\epsilon)$. For vacuum $B$, the paths are $A B, A B A B$, etc., so $P_{B}=P_{A} / \epsilon=$ $1 /(1-\epsilon)$. The vacuum $Z$ arises from paths $A B Z, A B A B Z$, etc., so $P_{Z}=(1-\epsilon) P_{B}=1$. To normalize, we divide by $P_{A}+P_{B}+P_{Z}=2 /(1-\epsilon)$, which yields

$$
p_{A}=\frac{\epsilon}{2}, \quad p_{B}=\frac{1}{2}, \quad p_{Z}=\frac{1-\epsilon}{2} .
$$

Now, suppose that the initial vacuum is $B$. The associated tree is shown on the right of Fig. 1. One finds $P_{A}=\epsilon /(1-\epsilon), P_{B}=P_{A}$, and $P_{Z}=1$. Hence the normalized probabilities are

$$
p_{A}=p_{B}=\frac{\epsilon}{1+\epsilon}, \quad p_{Z}=\frac{1-\epsilon}{1+\epsilon} .
$$



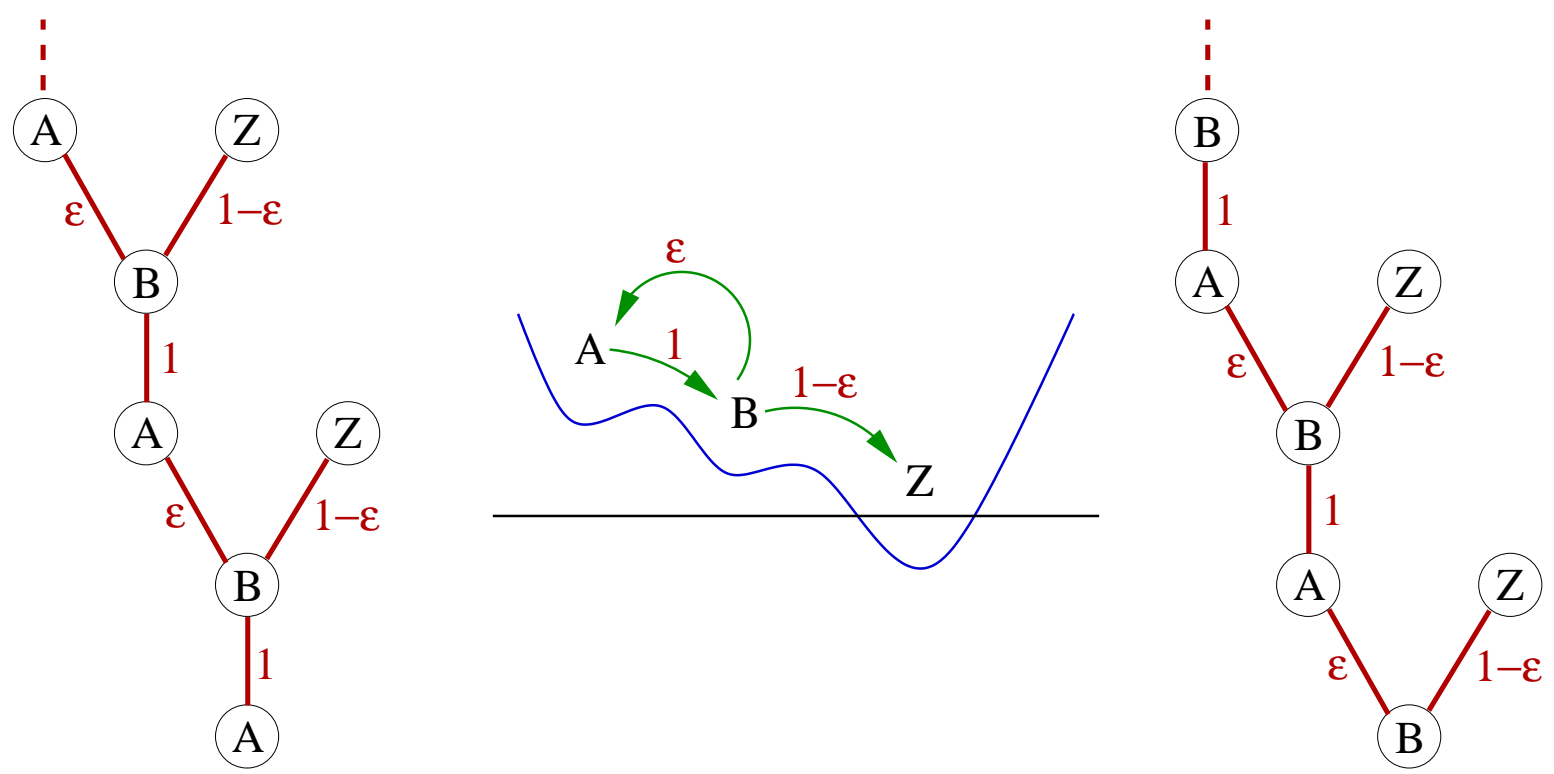

Figure 1: A landscape with two metastable vacua and one terminal vacuum. The tree on the left corresponds to a worldline starting in vacuum $A$ (the initial vacuum, or root). The tree on the right starts with vacuum $B$. The unnormalized probability for vacuum $i$ is obtained by computing the probability for each path leading up from the root to $i$ (the product of the numbers along the path), and summing over all paths.

As one would expect for a single worldline, the probability to pass through a given vacuum can depend strongly on the initial vacuum.

It is interesting to take note of the limiting values of the above probabilities as $\epsilon \rightarrow 0$, or $\epsilon \rightarrow 1$. These are physically the most relevant cases, because the rates for different decay channels generically differ by exponentially large factors.

\subsection{Convergence and pruned trees}

It is not obvious that the raw probabilities, Eq. (2.2), are finite. We will now show that the sum converges, if and only if the landscape constains at least one terminal vacuum. Moreover, we will show that trees can be significantly pruned without affecting normalized probabilities. When Eq. (2.2) is applied to the pruned tree it gives a finite answer in all cases. Hence, we shall take the pruned tree as our general prescription.

It is useful to think of the tree in terms of a conserved current (in this case, a probability current). A unit current enters the tree at the root (the source) and flows up the tree, ending up exclusively in terminal vacua (the sinks). It immediately follows 
that the total raw probability for all terminal vacua is unity:

$$
\sum_{z} P_{z}=1
$$

where the sum runs over terminal vacua. By the connectedness of the landscape, the probability $f$ to flow from a metastable vacuum $a$ to some terminal vacuum $z$ is nonzero. Since $P_{z} \geq f P_{a}$, it follows that all $P_{a}$ are finite. Hence, the sum in Eq. (2.2) converges for all $i$, if the landscape is terminal. (A proof for "if and only if" is given further below.)

The pruned tree is the smallest part of the full tree, such that the full tree consists of copies of the pruned tree joined together. Starting at the root, the pruned tree is constructed like the full tree, except that one terminates the tree wherever it returns to the initial vacuum $o$. For example, Fig. 2 shows pruned trees for the $A B Z$ model discussed above. Pruned trees need not be finite, however, as the example in Sec. 2.5 below will demonstrate.

Analogous to Eq. (2.2), one can compute unnormalized probabilities from a pruned tree:

$$
\mathcal{P}_{i}=\sum_{\begin{array}{c}
\text { all nodes } \\
\text { of the pruned tree } \\
\text { labelled } i
\end{array}} \begin{gathered}
\text { the branches } \\
\text { forming the path } \\
\text { from the root to the node }
\end{gathered}
$$

As in Eq. (2.6), the conservation of the probability current implies that

$$
\mathcal{P}_{o}+\sum_{z} \mathcal{P}_{z}=1
$$

Continuing the same argument as above, this implies that all raw probabilities computed from the pruned tree are finite. However, because $o$ is now effectively treated like a terminal vacuum, this conclusion applies independently of the presence of terminal vacua.

The full tree can be reconstructed from the pruned tree by joining a copy of the pruned tree at the root to every final node labeled $o$ in the original pruned tree, and iterating (see Fig. 2). This means that the raw probabilities of the full tree, $P_{i}$, will be given by

$$
P_{i}=\mathcal{P}_{i}\left(1+\mathcal{P}_{o}+\mathcal{P}_{o}^{2}+\ldots\right)=\frac{\mathcal{P}_{i}}{\sum_{z} \mathcal{P}_{z}}
$$

It follows that the raw probabilities computed from the full tree, $P_{i}$, converge if and only if there are terminal vacua. 


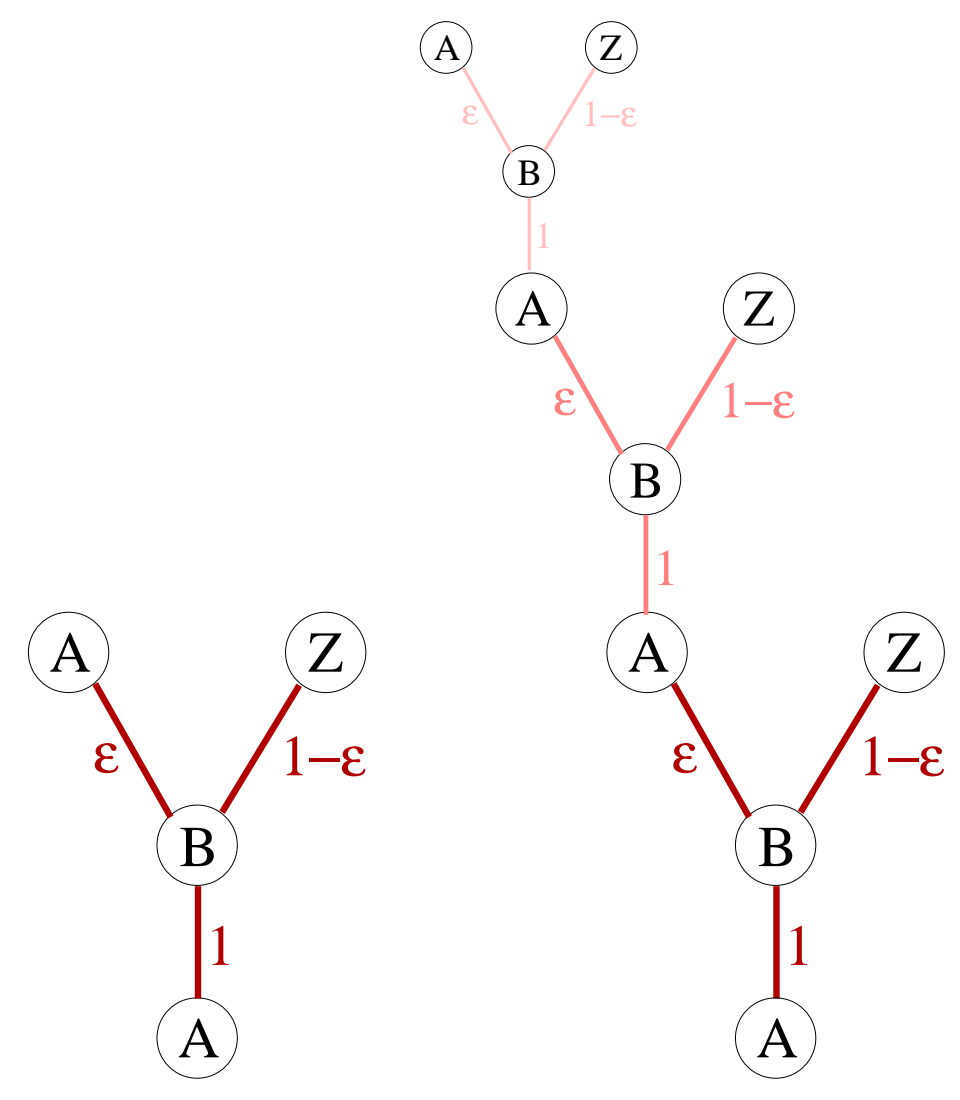

Figure 2: Probabilities are easier to compute from the pruned tree, shown left for the $A B Z$ model, with initial vacuum $A$. One reads off readily that $\mathcal{P}_{A}=\epsilon, \mathcal{P}_{B}=1$, and $\mathcal{P}_{Z}=1-\epsilon$, which need only be normalized. Right: The full tree can be recovered by iterating the pruned tree. Each iteration merely changes all raw probabilities by an overall factor, leaving the normalized probabilities unchanged.

In the terminal case, when the $P_{i}$ do converge, Eq. (2.9) implies that the vectors $\mathbf{P}$ and $\mathcal{P}$ are proportional. Hence the normalized probability,

$$
p_{i}=\frac{\mathcal{P}_{i}}{\sum_{j} \mathcal{P}_{j}}
$$

will agree with that computed from the full tree, Eq. (2.3).

Thus, from now on we will use the pruned tree as a basis for computing probabilities. Whenever the full tree gives well-defined results, they agree with those from the pruned tree. But the pruned tree is much simpler, and it yields well-defined probabilities even for cyclic landscapes.

\subsection{A cyclic example}

Consider a landscape with three metastable vacua $A, B$, and $C$, as shown in Fig. 3. In 
general, there would be transitions between all pairs of vacua. For simplicity, however, we will assume that $A$ and $C$ cannot decay into each other directly but only through $B$. Thus the model is very similar to the $A B Z$ model studied in Sec. 2.3, except that $C$ eventually decays back up to $B$.

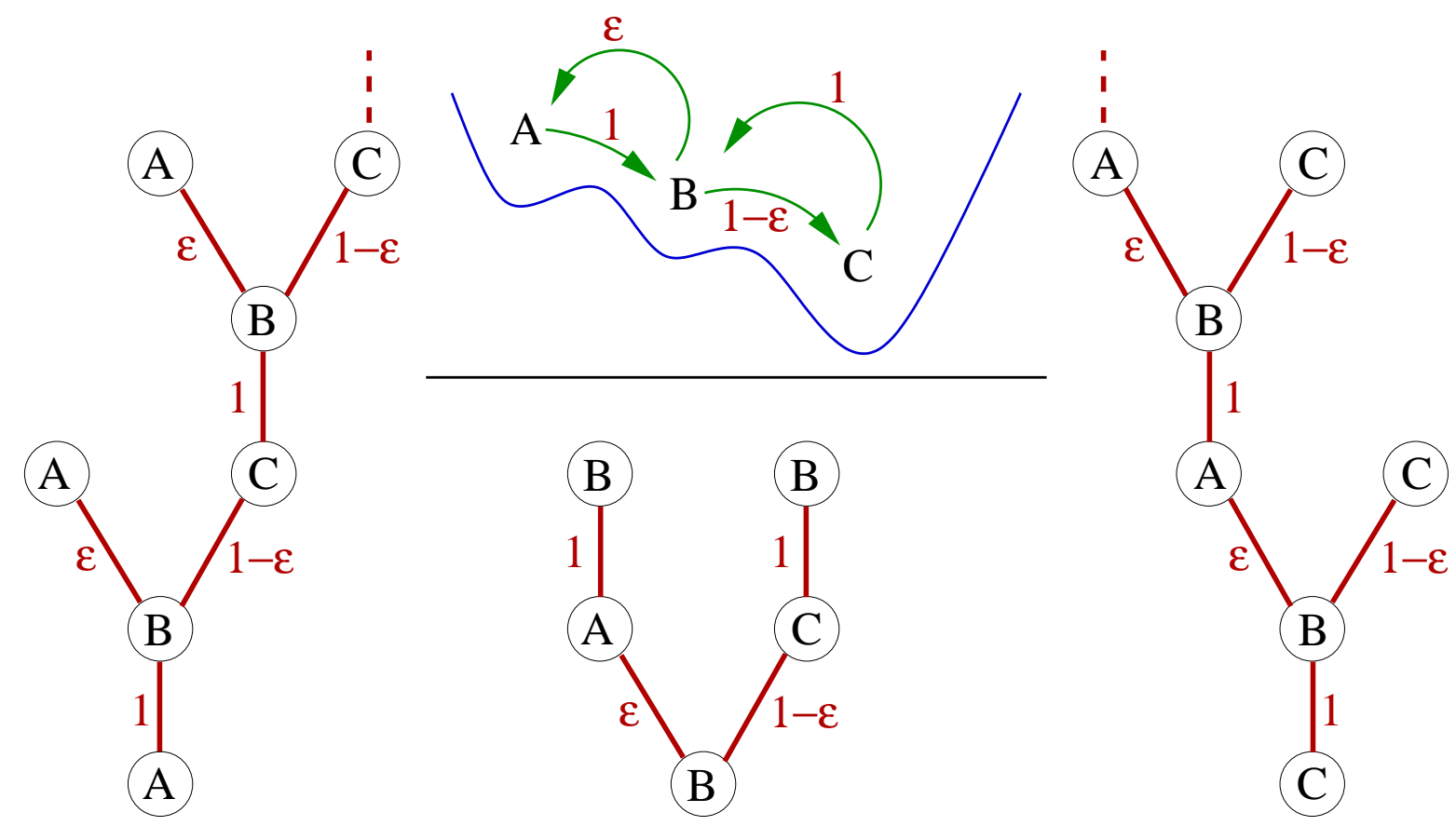

Figure 3: A landscape without terminal vacua. For each initial vacuum, a pruned tree is shown. For example, summation over paths in the left tree yields $\mathcal{P}_{A}=1, \mathcal{P}_{B}=1 / \epsilon$, $\mathcal{P}_{C}=(1-\epsilon) / \epsilon$. After normalization, all pruned trees yield the same probabilities.

The pruned tree will depend on the initial vacuum (see Fig. (3). One easily checks, however, that they all yield the same normalized probabilities:

$$
p_{A}=\frac{\epsilon}{2}, \quad p_{B}=\frac{1}{2}, \quad p_{C}=\frac{1-\epsilon}{2} .
$$

Note the special cases $\epsilon \rightarrow 0, \epsilon=1 / 2$, and $\epsilon \rightarrow 1$. In the next subsection, we will prove that $p_{i}$ is always independent of the initial condition in a cyclic landscape.

\subsection{Matrix formulation}

We have seen that graphs provide an intuitive and general method of defining probabilities. Let us reformulate the result as a matrix equation. This is natural since $\eta_{i j}$ forms a matrix $\eta$ and the raw probabilities $P_{j}$ form a vector $\mathbf{P}$.

For any tree, we can define the partial probability $P_{i}^{(\alpha)}$ as the probability to reach vacuum $i$ after $\alpha$ steps from the initial vacuum. It will be convenient to define the initial 
probability vector $\mathbf{P}^{(0)}$, which satisfies $P_{j}^{(0)}=1$ for $j=o$ and $P_{j}^{(0)}=0$ otherwise. (Here we continue to assume that the worldline starts out in one vacuum $o$. Our result, Eq. (2.14) below, will naturally incorporate more general initial probability distributions $\mathbf{P}^{(0)}$.)

On a full tree, the partial probabilities obey

$$
\mathbf{P}^{(\alpha)}=\eta \mathbf{P}^{(\alpha-1)}
$$

The raw probability is the sum of partial probabilities:

$$
\mathbf{P}=\sum_{\alpha=1}^{\infty} \mathbf{P}^{(\alpha)}
$$

The above two equations imply that the raw probability obeys the matrix equation

$$
(1-\eta) \mathbf{P}=\eta \mathbf{P}^{(0)}
$$

To be consistent with the results of Sec. 2.4, this equation should have a solution if and only if the landscape is terminal. Let us prove this. Suppose that there is no solution. Then $(1-\eta)$ cannot be invertible, and $\eta$ must have an eigenvalue 1 with eigenvector $\tilde{\mathbf{P}}$, satisfying $\tilde{\mathbf{P}}=\eta \tilde{\mathbf{P}}$. We are free to think of $\tilde{\mathbf{P}}$ as a partial probability, in which case this describes an equilibrium: the probability distribution is unchanged by an extra step. By connectedness of the landscape, this implies that the landscape contains no terminal vacua. Conversely, suppose that the landscape has no terminal vacua. This means that for any nontrivial initial condition $\mathbf{P}^{(0)} \neq 0$, the vector $\eta \mathbf{P}^{(0)}$ must have some nonzero components, and in particular, their sum is nonzero. It also means that every column of $\eta$ adds up to 1 , so the components of $(1-\eta) \mathbf{P}$ add to zero. Thus, Eq. 2.14) cannot be solved.

To deal with both the terminal and cyclic cases, we used pruned trees, which are obtained by treating the vacuum $o$ as a terminal vacuum, except in the first step. Thus, pruned trees obey the matrix equation

$$
(1-\eta S) \mathbf{P}=\eta \mathbf{P}^{(0)}
$$

where $S$ annihilates the $o$-th column of $\eta$ :

$$
S_{i j}=\delta_{i j}-P_{i}^{(0)} P_{j}^{(0)}
$$

By the above proof, $(1-\eta S)$ is invertible so Eq. (2.15) always has a unique solution. Thus, it is the most general matrix equation we shall require. However, in the terminal case, Eq. (2.14) is equivalent and more elegant. 
In fact, a simpler specialized equation is also available in the cyclic case:

$$
(1-\eta) \mathbf{P}=0
$$

This follows because the pruned tree will have $P_{o}=P_{o}^{(0)}$ by conservation of the probability current; see Eq. (2.8). Hence

$$
\eta S \mathbf{P}+\eta \mathbf{P}^{(0)}=\eta\left(S \mathbf{P}+\mathbf{P}^{(0)}\right)=\eta \mathbf{P} .
$$

Substitution into Eq. (2.15) yields Eq. (2.17).

Note that $\mathbf{P}^{(0)}$ does not appear in Eq. (2.17). This demonstrates that the probabilities are independent of the initial vacuum in the cyclic case, as advertised above.

To avoid confusion, let us emphasize once more that the general ("pruned tree") prescription is captured by Eq. (2.15). It reduces to Eqs. (2.14) and (2.17) for terminal and cyclic landscapes, respectively.

In Ref. [13] the general prescription of Garriga et al. [7] was applied to the special case of cyclic landscapes (for the simplest cyclic landscape the result was first given in Ref. [15]). The probabilities obey Eq. (2.17), which shows that our prescription agrees with that of Garriga et al., if the landscape is cyclic. For terminal landscapes, such as the string landscape, the two prescriptions differ.

\section{Weighting}

In this section we ask with what probability $w_{i}$ observers will emerge in vacuum $i$. The total probability for $i$ to be observed is $p_{i} w_{i} / \sum_{j} p_{j} w_{j}$.

Anthropic arguments make sense only in a large and varied universe, where they select for location (rather than for initial conditions, or worse, for parameters of a fundamental theory). With a much larger cosmological constant but all other physics fixed, for example, it is plausible that life would not have formed in our part of the universe [19].

The problem is that other parameters are far from fixed in any realistic landscape. This poses a hard optimization problem, requiring variations of the possible inflaton potentials [20,21], particle and force content [22], coupling constants, and other parameters. Moreover, the challenge of identifying conditions for "life" will be magnified, if the landscape contains low energy theories so different from our own that we have little intuition for their impact on cosmology or condensed matter physics.

But whatever observers may consist of, they must obey the laws of causality, thermodynamics, and information theory. Observers compute; they store and retrieve information. This suggests that the more number of bits are contained in a causally 
connected region, the more likely it is that observers will emerge. Entropy bounds limit this number in terms of the maximal area on the future boundary of the causal diamond [10].

For example, in a de Sitter vacuum, the maximal entropy is $3 \pi / \Lambda$; if we ignore black holes, the bound goes down to about $\Lambda^{-3 / 4}$. From this point of view, a small cosmological constant is better than a large one, but because no other parameters are held fixed, there is no sharp Weinberg cutoff. ${ }^{4}$ Moreover, the preference for small $\Lambda$ is only power-law, not exponential as a purely statistical argument would have it.

But it is not enough to maximize for entropy in the causal diamond. For example, if the de Sitter vacuum starts out empty, or filled with nothing but thermal radiation, it is unlikely that any observers will emerge. The problem is that there is no free energy; the entropy is already maximal to start with. Thus, what we seek is a large entropy difference (the entropy leaving through the top cone of the diamond minus the entropy entering the bottom cone of the diamond).

This is presumably only a necessary condition, and there may be additional conditions or refinements (in particular, if there are stable vacua with $\Lambda=0$, a case where the entropy difference diverges). But this simple consideration already has a number of interesting consequences.

For example, it may seem natural that inflationary volume factors (which are welldefined in noneternal slow-roll) should enter directly into the weight factor [7,21]. But from a holographic point of view, volume produced in excess of one causally connected region does not boost the likelihood of a vacuum further. Inflation is useful in that it delays the time of curvature domination, allowing more free energy to be harvested. But there is no benefit in making that time longer than $|\Lambda|^{-1 / 2}$, the time when the cosmological constant begins to dominate.

Similarly, one may be tempted to include the lifetime of a metastable vacuum in its weight. But lifetime matters only up to a point. If the decay disrupts the harvesting of free energy, it will enter the weight factor. However, in many cases the region will thermalize long before it decays. Observers are likely to emerge only while there is a free energy gradient. Hence, excessive stability does nothing to boost their chances.

The production of free energy tends to be associated with bubble nucleation events. This is why it was appropriate for our probability measure to count repeated nucleations of the same vacuum separately. A possible complication is that the free energy produced may depend on the parent vacuum. This can be incorporated in our prescription by giving different labels to the same vacuum, depending on the amount of free energy produced, computing the prior probabilities, and weighting.

\footnotetext{
${ }^{4}$ G. Perez has independently arrived at this conclusion.
} 
In the holographic viewpoint, the maximum entropy can be regarded as a firm upper limit on the weight. The weight can be smaller, for example if the entropy is nonzero to start with, but it cannot be increased by factors coming from inflationary expansion or lifetimes. Our vacuum has a positive cosmological constant, so its weight is bounded. Suppose that the landscape were infinite, in the sense that parameters could scan arbitrarily dense discretua. Then it would be surprising that we should not find ourselves in a region that allowed for even greater complexity than our own. The landscape must be finite, and numbers such as $10^{-123}=e^{-283.2}$ [23] may turn out to be data points that will help us determine its size empirically.

Acknowledgements I would like to thank B. Freivogel, L. Hall, S. Kachru, A. Linde, A. Maloney, G. Perez, J. Polchinski, E. Silverstein, S. Shenker, L. Susskind, T. Watari, and I. Yang for discussions. This work was supported by the Berkeley Center for Theoretical Physics, by a CAREER grant of the National Science Foundation, and by DOE grant DE-AC03-76SF00098.

\section{References}

[1] R. Bousso and J. Polchinski: Quantization of four-form fluxes and dynamical neutralization of the cosmological constant. JHEP 06, 006 (2000), hep-th/0004134.

[2] S. Kachru, R. Kallosh, A. Linde and S. P. Trivedi: De Sitter vacua in string theory. Phys. Rev. D 68, 046005 (2003), hep-th/0301240.

[3] L. Susskind: The anthropic landscape of string theory (2003), hep-th/0302219.

[4] F. Denef and M. R. Douglas: Distributions of flux vacua. JHEP 05, 072 (2004), hep-th/0404116.

[5] A. Linde, D. Linde and A. Mezhlumian: Nonperturbative amplifications of inhomogeneities in a self-reproducing universe. Phys. Rev. D 54, 2504 (1996), gr-qc/9601005.

[6] J. Garriga and A. Vilenkin: A prescription for probabilities in eternal inflation. Phys. Rev. D 64, 023507 (2001), gr-qc/0102090.

[7] J. Garriga, D. Schwartz-Perlov, A. Vilenkin and S. Winitzki: Probabilities in the inflationary multiverse. JCAP 0601, 017 (2006), hep-th/0509184.

[8] R. Easther, E. A. Lim and M. R. Martin: Counting pockets with world lines in eternal inflation (2005), astro-ph/0511233.

[9] T. Banks and M. Johnson: Regulating eternal inflation (2005), hep-th/0512141. 
[10] R. Bousso: Positive vacuum energy and the N-bound. JHEP 11, 038 (2000), hep-th/0010252.

[11] L. Susskind, L. Thorlacius and J. Uglum: The stretched horizon and black hole complementarity. Phys. Rev. D 48, 3743 (1993), hep-th/9306069.

[12] R. Bousso, B. Freivogel and I.-S. Yang: to appear.

[13] V. Vanchurin and A. Vilenkin: Eternal observers and bubble abundances in the landscape (2006), hep-th/0605015.

[14] L. Dyson, M. Kleban and L. Susskind: Disturbing implications of a cosmological constant. JHEP 10, 011 (2002), hep-th/0208013.

[15] R. Bousso, B. Freivogel and M. Lippert: Probabilities in the landscape: The decay of nearly flat space (2006), hep-th/0603105.

[16] E. Farhi and A. H. Guth: An obstacle to creating a universe in the laboratory. Phys. Lett. B183, 149 (1987).

[17] R. Bousso: Cosmology and the S-matrix. Phys. Rev. D 71, 064024 (2005), hep-th/0412197.

[18] D. Green, E. Silverstein and D. Starr: Attractor explosions and catalyzed vacuum decay (2006), hep-th/0605047.

[19] S. Weinberg: Anthropic bound on the cosmological constant. Phys. Rev. Lett. 59, 2607 (1987).

[20] M. Tegmark and M. J. Rees: Why is the CMB fluctuation level $10^{-5}$ ?. Astrophys. J. 499, 526 (1998), astro-ph/9709058.

[21] B. Feldstein, L. J. Hall and T. Watari: Density perturbations and the cosmological constant from inflationary landscapes. Phys. Rev. D 72, 123506 (2005), hep-th/0506235.

[22] R. Harnik, G. D. Kribs and G. Perez: A universe without weak interactions (2006), hep-ph/0604027.

[23] J. Polchinski: The cosmological constant and the string landscape (2006), hep-th/0603249. 